\title{
When is electromagnetic spectrum fungible?
}

\author{
Martin Weiss \\ School of Information Sciences \\ University of Pittsburgh \\ Pittsburgh, Pennsylvania 15260 \\ Email: mbw@pitt.edu
}

\author{
Prashant Krishnamurthy \\ School of Information Sciences \\ University of Pittsburgh \\ Pittsburgh, Pennsylvania 15260 \\ Email: prashk@pitt.edu
}

\author{
Linda E. Doyle \\ CTVR \\ Trinity College \\ Dublin, Ireland \\ Email: ledoyle@tcd.ie
}

\author{
Konstantinos Pelechrinis \\ School of Information Sciences \\ University of Pittsburgh \\ Pittsburgh, Pennsylvania 15260 \\ Email: kpele@pitt.edu
}

\begin{abstract}
Fungibility is a common assumption for marketbased spectrum management. In this paper, we explore the dimensions of practical fungibility of frequency bands from the point of view of the spectrum buyer who intends to use it. The exploration shows that fungibility is a complex, multidimensional concept that cannot casually be assumed. We develop two ideas for quantifying fungibility - (i) of a fungibility space in which the "distance" between two slices of spectrum provides score of fungibility and (ii) a probabilistic score of fungibility.
\end{abstract}

\section{INTRODUCTION}

Secondary markets for radio spectrum are seen as a step toward continuous reassignment of radio spectrum to its highest purpose and to enable correct valuation of wireless service providers [1]. The fungibility of electromagnetic spectrum has been broadly recognized to be a requirement of feasible spectrum markets [1]-[6]. At the same time, conventional wisdom among researchers and policymakers questions whether the fungibility requirement holds in practice. In order to shed light on this, we examine the question of fungibility of radio spectrum in this paper.

Electromagnetic spectrum is valuable because it enables capabilities that increase social welfare. These capabilities usually involve purposeful communications that may be unidirectional or bidirectional; for the purpose of this paper, we assume that this is the case. The question then arises as to what exactly is the value of a slice of electromagnetic spectrum? Is one slice exactly identical to another slice of the same size, but at a different frequency (i.e., will it provide the same communication capabilities)? While for example, Kwerel and Williams assert that frequencies in the $300 \mathrm{MHz}$ to $3000 \mathrm{MHz}$ band are "approximately fungible" [4], more recent research indicates different auction prices obtained for different frequencies in this band [7] in practice, indicating that the economic value is not the same for different slices of spectrum. Outside of this economic evidence, we examine technical and practical issues related to the fungibility of spectrum, such as the allowed transmit power and physical layer alternatives, the propagation characteristics at different frequencies, the interference features, the capabilities of the communicating devices, and the application needs since they all influence the communication capabilities afforded by a slice of spectrum at a specific frequency.

Our objective is to record the factors influencing fungibility. Such an examination and characterization would be beneficial in modeling the value of slices of electromagnetic spectrum, perhaps in secondary markets. The exploration shows that fungibility is a complex, multidimensional concept that cannot casually be assumed. We develop two ways of determining spectrum fungibility - (i) using the idea of a fungibility space in which the "distance" between two slices of spectrum provides the fungibility score and (ii) a probabilistic fungibility score reflecting the likelihood of two bands being fungible. The rest of the paper is organized as follows. In Section II, we define the term "fungible" both economically and as it pertains to radio systems. In Section III, we qualitatively describe the limits of fungibility of spectrum. Section IV translates these limits into reality based on the available research literature. Section V concludes the paper.

\section{BACKGROUND}

\section{A. Economic definitions of fungibility}

Merriam-Webster defines "fungible" as

"being of such a nature that one part or quantity may be replaced by another equal part or quantity in the satisfaction of an obligation."

The main idea here is that of substitutability. When commodities are fungible, one instance of an item can replace another instance of that item "in the satisfaction of obligation". Such an obligation may simply be a trade or commitment to buy or sell. This suggests that one instance of a commodity is of equal value to the other instance of the commodity. In physical commodities, we might say that gold is fungible because any ounce of gold can substitute for any other ounce of gold as long as the purity and weight of each ounce meets the same standards. In physical commodities, such as metals, grains, meats, etc., fungibility is obtained by defining standard trading units [8]. Thus, when traders buy or sell a commodities contract, they have a clear understanding of what they are buying or selling even if they never inspect the item.

\section{B. Importance of fungibility}

Fungibility is important in economic transactions because it leads to lower transaction costs, which, in turn, leads to markets (as an organizational form) [9]. When goods are not fungible, markets are unlikely to emerge because they cannot allocate transaction costs efficiently. Non-fungible goods often lead to vertically integrated systems (i.e., hierarchies) which 
poses some challenges to the valuation and dynamic change in the wireless industry.

\section{Operational definitions for radio systems}

Doyle and Forde [3] summarize the approaches taken over time in an effort to define property rights in spectrum. Weiser and Hatfield [10] discuss the challenges of defining these property rights, and Hazlett [11] argues that adjudication is possible despite these challenges. Weiss and Liu [12] propose an alternative to the usage rights paradigm through markets in interference rights.

Despite these debates, there is general agreement that spectrum rights, to the extent that they may be tradeable, must be defined in space, time and frequency at the very least. We elaborate on this in later sections.

\section{Economic evidence of spectrum trades}

Fungibility does not appear to drastically affect the volume of spectrum trades in the US: Mayo and Wallsten [13] examined the FCC's Universal Licensing System database and discovered that trades were hardly uncommon across a variety of service bands. It is, however, important to note that this may not be generalized to other countries, as is clear from Akalu's work [2], [14]. Since the regulatory agencies do not publicize prices, it is not possible to build a secondary market demand curve by frequency. In fact, the trades in the US and the UK do not use exchange markets, but rather brokers (e.g., SpectrumBridge, Cantor Fitzgerald, etc.).

\section{E. Who makes the determination of fungibility?}

It is a maxim in markets that an asset is worth what someone will pay for it. More generally, in a (voluntary) market exchange, a trade can take place when the price is above the seller's reservation price and below the buyer's willingness-to-pay.

We assume that a buyer acquires spectrum because it enables him/her to accomplish a valuable function; thus, we exclude speculative spectrum purchases. Consequently, it is the buyer of spectrum that determines whether a frequency band is sufficiently equivalent to another (i.e., fungible) for their particular requirement.

Nevertheless, from the standpoint of quantifying fungibility, the lack of markets and a standardized unit of spectrum (save that of bandwidth) implies that we have to resort to known technical and regulatory factors as potential metrics as discussed below.

\section{ON The FUnGIBILITY OF "NAKed Spectrum"}

User demands take place at a particular point in time and point (region) in space. A system to meet user demands or needs must be able to provide communication capability to that point in space at that time, usually through one or more other points in space (i.e., system architecture components such as access points or base stations). Thus, we can conclude that communications demand is a (potentially complex) spatiotemporal phenomenon, so spectrum demand for purposeful use is also a complex spatio-temporal phenomenon. Fungibility, therefore, is limited in space and time. Further, technological and regulatory issues complicate fungibility determination as described below.

In this section, we explore in modest detail the factors that would affect a buyer's decision about whether a particular offered frequency band is a reasonable substitute for another (i.e., is fungible). The object to be traded is what Hazlett has referred to as "naked spectrum", that is, frequency bands only with no infrastructure supporting it [15]. Technologically, this discussion is deliberately superficial, focusing only on the main factors that influence fungibility. The authors are aware that the technological alternatives are far richer than presented here, and that these present tradeoffs that could influence a fungibility determination one way or the other.

\section{A. Spatial}

In space, the coverage of a signal depends on the carrier frequency. To illustrate, let us assume a very simple free-space path-loss model where the path-loss $L_{p}$ is given by:

$$
L_{p}=21.98-20 \log _{10}(c / f)+20 \log _{10} d
$$

where $c$ is the speed of light, $f$ is the frequency of the carrier, and $d$ is the distance between the transmitter and receiver. Even under such a simple model, for the same transceiver features (transmit power and receiver sensitivity), clearly the coverage d depends on the frequency $f$. As shown in Figure 1, the coverage varies significantly with frequency. If coverage is the primary criterion, then spectrum becomes fungible in one direction - a slice at a lower frequency can always be used by someone who would have been willing to use a slice at a higher frequency (again, site specificity may still have an impact).

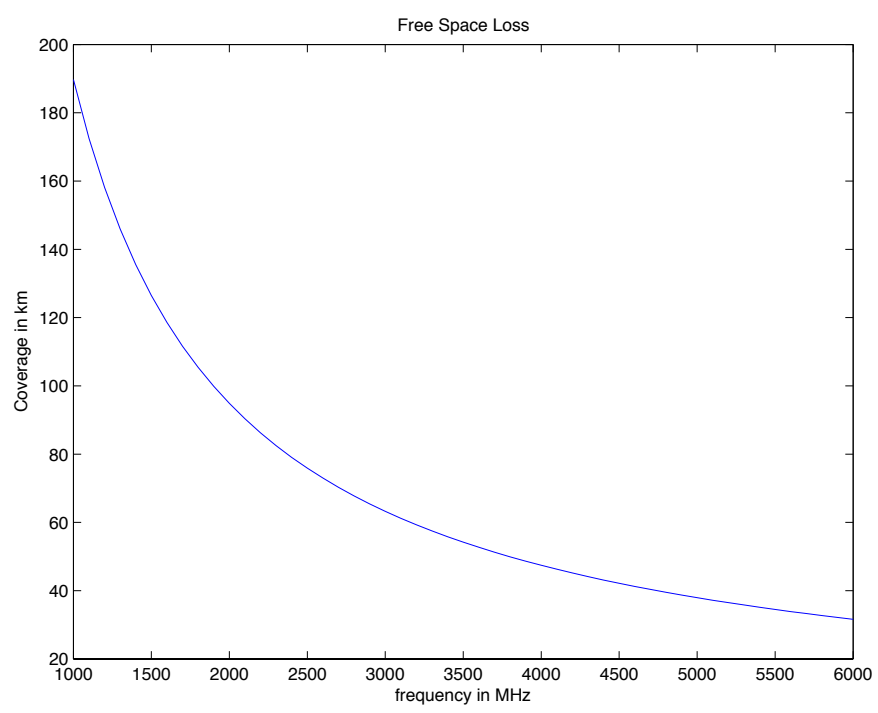

Fig. 1. Coverage at different frequencies assuming free-space loss when transmit power is $4 \mathrm{~W}$ and minimum acceptable received power is $-80 \mathrm{dBm}$

In architecture, the utility of a particular frequency band can depend on its fit to the existing infrastructure. For example, 
as Figure 1 illustrates, coverage for systems built for one frequency band, say $1500 \mathrm{MHz}$ may not be able to provide full coverage at, say, $2500 \mathrm{MHz}$. This does not mean that these two frequencies are not fungible, simply that the existing infrastructure may not suit all user demands if $2500 \mathrm{MHz}$ were used. Further, some of the coverage effects can be to some extent mitigated by investments in high gain antennas and higher transmission power (within economic and regulatory, constraints, of course). We observe that it would still be up to the buyer to determine if the higher frequency of 2500 $\mathrm{MHz}$ would meet the coverage requirement. If it did, spectrum at these two frequencies could be thought of as fungible (assuming other factors discussed below are not important) for the buyer's application. If it did not, then they would not be fungible.

Alternately, fungibility could be a function of price. Kerans et.al. [7] showed that the coverage differences described above affect prices in the primary (auction) market outcomes. In part, the differences in auction prices are due to the larger infrastructure investment requirements to cover the same area with higher frequency channels. It is reasonable to assume that they would affect outcomes in the secondary (trading) markets as well. That is, if a band is sufficiently cheap, a buyer might consider it fungible with another, more costly band that may meet his or her technical or operational requirements better.

\section{B. Temporal}

An example of how the availability or usefulness of spectrum changes over time is shortwave radio communications. Transmissions at certain frequencies in the shortwave bands are reflected by the ionosphere only at night allowing long range communications then, but not during the day. Another example of temporal variations is the load in cellular telephone networks. As noted in [16], the load in a cell varies significantly with time of day and day of week. For example, downtown areas may see significant loads during the day and light or no traffic at nights. In [16], the authors use this feature to dim cell sites to save energy. It is possible to allow secondary access to unused spectrum in the cellular bands when there is little usage. In fact, it may be possible to simply use the same cellular network infrastructure for secondary applications, especially with the ability of today's smart phones to perform a variety of generalized tasks, making this spectrum really attractive. This however assumes that such applications need spectrum only when the usage by the primary license holder is negligible. Similar temporal variations in availability of spectrum is discussed in [17].

\section{Technological}

There are several technological factors that impact fungibility. We consider a few of the most common factors below.

Systems that require two way communications must choose a form of duplexing. In general, there are two kinds of duplexing: Frequency Division Duplexing (FDD) and Time Division Duplexing (TDD). In FDD, the uplink and downlink channels are separated in frequency. By contrast, TDD systems use a single channel that is alternately uplink and downlink. Implementation tradeoffs abound and there is a literature comparing these approaches and evaluating the tradeoffs (see [18] and [19], for example). From the point of view of fungibility, one key factor is that an FDD system requires spectrum that is paired because the uplink and the downlink operate at different frequencies. Further, these have to be sufficiently far apart for cost effective systems with minimal crosstalk between the uplink and downlink (45 MHz for GSM systems). Thus, an FDD system needs a pair of channels that are sufficiently far apart (but not too far) that meet the spatio-temporal coverage requirements discussed above to be considered fungible with another pair of channels.

The air interface can also be a factor. FDMA systems are in some senses the easiest to consider, since the purchaser would acquire a clear spectrum band. TDMA and CDMA systems require much closer coordination between primary and secondary users, which means that a candidate band would only be suitable if the air interface was compatible with the buyers system. That is a spectrum opportunity in a band where the operator is using TDMA band may not be fungible with an otherwise similar band in frequency, etc. for a system using CDMA.

Finally, the usage of spectrum slices adjacent to the ones under consideration and the applications they support may impact what could be done with the spectrum. That is, a key question is compatibility with neighboring systems in the band that is being considered. For example, as widely reported over the last couple of years [20], the company Lightsquared has rights to the spectrum in the range of 1525-1559 $\mathrm{MHz}$. However, it was unable to employ this spectrum for terrestrial mobile broadband services because of the harmful interference it would cause to navigation and precision positioning services employing the 1559-1610 MHz slices. While satellite services at low power in the $1525-1559 \mathrm{MHz}$ spectrum were still possible, other applications would not be easily feasible reducing the value of this spectrum. Additional costs of upgrading navigational receivers would only solve part of the problem, as precision positioning would still be impacted.

\section{Regulatory and Other Issues}

Finally, the intended purpose of the spectrum (i.e., its allocation) and regulatory considerations can also play a role. For example the necessary throughput and delay can affect the choice of spectrum. Regulatory requirements may limit the use of certain bands to certain applications, modulation schemes, power levels, etc. (e.g., the AM band may only be used for broadcasting-related purposes, with power and modulation limitations as specified by the national regulatory authority).

\section{TOWARDS A "FungibILITy SCORE"}

The fungibility requirement thus has spatial, temporal, technological, and regulatory dimensions as discussed above. Spatial aspects are determined by the antenna type and placement, permitted transmission power and electromagnetic propagation 
characteristics. Temporal dimensions are determined by the needs of the application and the availability of the band; this is especially relevant in time limited secondary use situations. The technological dimensions can include duplexing (FDD or TDD), air interface, modulation, etc. The regulatory dimension considers the possibility of using a spectrum slice for a particular application.

The question that we tackle next is to quantify fungibility in a meaningful manner. In [1], the authors propose a "viability score" to assess the general viability of alternative approaches to spectrum trading markets. The use of such a score enabled them to combine a variety of measures into a single metric that enabled comparison across scenarios. As we discussed above, a determination of fungibility is a comparative exercise that is conducted by the (potential) buyer. Buyers are evaluating whether spectrum opportunity $\mathrm{A}$ is substitutable for spectrum opportunity B with respect to their spatio-temporal and technological requirements. From the standpoint of the secondary user, then, the important questions for both spectrum opportunities are:

- Can I tune my radios to the target band?

- Does the target band meet my minimum throughput requirements?

- Does the target spectrum opportunity meet my duplexing requirements?

- Does the target spectrum opportunity meet my minimum spatial coverage requirements?

- Can I support the applicable air interface requirements?

All of these can be viewed simply as binary variables based on minimum requirements, but it may prove to be less useful to end users if we provide only a binary answer as to whether two slices of spectrum are fungible. More quantitative answers to these questions can be derived from objective characteristics of the target spectrum, including: frequency band, bandwidth, geographic area, duplex type, and technical and regulatory limitations. Because of the stochastic nature of electromagnetic propagation, some of these properties (notably throughput and coverage) are not deterministic. The answers to the questions also depend on the location(s) of the secondary user's infrastructure. So, in general, we do not see the fungibility of two frequency bands as absolute (or with $100 \%$ probability).

Instead, we believe that we can only speak of two bands being fungible along a continuum, defined by a fungibility score. We consider two different ways of obtaining this score. The first approach looks at two bands "being fungible with probability $x$ for user $y$ and application $z$ ". The fungibility score could then be this probability. Considering each of the dimensions as independent, the overall fungibility score would be a product of the probabilities. The second approach looks at a normalized "distance" between two bands in a multidimensional fungibility space and quantifies the score through this distance. In computing "distance" between two bands, it becomes necessary to "weight" the distance or normalize the distance so that one particular dimension does not always overwhelm the other fungibility dimensions; alternatively, the weight of a dimension can reflect the relative importance of the dimension in the eyes of the buyer.

What we are proposing here is not an absolute measure of fungibility, but a framework to evaluate fungibility that can evolve with information about additional parameters in each category. In doing so, it is important to recognize that the structure of a metric depends on what it is used for. For example, a spectrum broker is interested in fungibility, not because s/he is buying it, but because they need to market and price available frequency bands (or bundles of bands) in particular markets to potential buyers. Alternatively, spectrum buyers may be interested in sorting or prioritizing bands for consideration for their system application. These differing objectives could result in differing approaches to developing a fungibility score.

We propose two such approaches here, fully aware that others may exist as well. We refer to them as the "probabilistic" and the "distance-based" approaches. The former is not necessarily probabilistic in the technical sense, though a spectrum broker might use it as a measure for the likelihood of making a trade. The second approach uses a Euclidean distance strategy to assess how "far" two spectrum opportunities are away from each other. A buyer may use this to prioritize spectrum opportunities.

\section{A. Probabilistic and Distance-based Fungibility Scores}

Next we consider the calculation of the fungibility score using these two approaches in various dimensions. We will assume in what follows, that the spectrum that a user's need is centered around $f_{1}$ with a bandwidth of $W_{1} \mathrm{~Hz}$ while the spectrum that is being considered as a (fungible) option is centered around $f_{2}$ with a bandwidth of $W_{2} \mathrm{~Hz}$. Unless otherwise mentioned, we will assume that $W_{1}=W_{2}$.

a) Spatial: In the spatial dimension, there are still many variables that have to be accounted for. First, the transmit powers may be variable depending on the regulatory constraints. Second, the available/necessary infrastructure to provide coverage may be different. For instance, multiple base stations may still be able to provide coverage while employing a lower power or higher frequency (which typically has higher pathloss). The environment-specific issues have to be considered in the computation of path-loss. To keep the computation simple, in this preliminary work we only consider point-to-point-like links in free space with the same transmit power and receiver capabilities for comparing slices of spectrum.

Probabilistic Score: We can now use the fraction of coverage provided by the alternative slice of spectrum as the unit for the probabilistic score. In this case, the fraction of coverage provided can be determined easily as follows. With the transmit powers and receiver capabilities the same, the allowable pathloss has to be the same in both systems for communication links to be created. Thus, from (1):

$$
\begin{aligned}
& \log _{10} \frac{d_{2}}{d_{1}}=\log _{10} \frac{f_{1}}{f_{2}} \\
& \Rightarrow P_{\text {spatial }}=\min \left(\frac{d_{2}}{d_{1}}, 1\right)=\min \left(\frac{f_{1}}{f_{2}}, 1\right)
\end{aligned}
$$


where $P_{\text {spatial }}$ is the probabilistic score. We note here that a frequency $f_{2}<f_{1}$ may provide greater coverage than necessary, in which case, we assume that $100 \%$ coverage is available.

Distance Score: For the purposes of this paper, we would like to constrain the distance to be between 0 and 1 . For that reason, we pick the following distance metric. Let $d_{1}\left(d_{2}\right)$ be the coverage obtained using $f_{1}\left(f_{2}\right)$ with a given transmit power and receiver capability in free space. The distance $D_{\text {spatial }}$ in this dimension would be:

$$
D_{\text {spatial }}=\frac{\max \left(d_{1}-d_{2}, 0\right)}{d_{1}}
$$

We note here that if $f_{2}=f_{1}$, i.e., we are comparing a band with itself, the probabilistic score would be $P_{\text {spatial }}=1$ and the distance score would be $D_{\text {spatial }}=0$.

b) Temporal: As is the case with the spatial dimension, the temporal dimension has many variables. For simplicity, we will only consider the availability $T_{f_{2}, a v}$ of the band at $f_{2}$ over a fixed unit of time $T$ for which the band at $f_{1}$ is available. If $f_{2}$ is not available completely for this duration, the fraction of time that it is available will be used as the metric for the probabilistic score and the normalized difference in duration will be the distance, i.e.,

$$
\begin{aligned}
P_{\text {temporal }} & =\frac{\min \left(T_{f_{2}, a v}, T\right)}{T} \\
D_{\text {temporal }} & =\frac{\max \left(T-T_{f_{2}, a v}, 0\right)}{T}
\end{aligned}
$$

Note if $T_{f_{2}, a v} \geq T, P_{\text {temporal }}=1$ and $D_{\text {temporal }}=0$.

c) Technological: This is perhaps the most complex dimension to be quantified and may vary on a case-by-case basis. In other words, it is not easy to provide general expressions as is the case of spatial and temporal availability. We acknowledge that in this paper we do not rigorously capture the various issues in the technological dimension. In fact, we only consider the technological dimension in a heuristic way. There is however the potential to include reasonable, yet simple models to account for various technological factors. We enumerate our heuristic approach next for a few technological issues.

1) Duplexing: We will assume a cost for time division duplexing when the preference is for a paired channel. Assuming that the bandwidth is the same, we assign a probabilistic score of 0.5 (i.e., an unpaired channel has a $50 \%$ chance of being suitable for the application) or a distance score that is also 0.5 (an unpaired channel is not completely unsuitable, but it is not completely compatible either).

2) Frequency Tunability: With the increased sophistication of technology, frequency tunability is becoming less of an issue for most devices. For example, as far back as 2008, Broadcom had integrated 802.11n, Bluetooth, and FM (both transmission and reception) into its BMC4329 chip [21]. Most smartphones of today can support a variety of frequency bands for cellular communications (ranging from $500 \mathrm{MHz}$ to $3 \mathrm{GHz}$ ), a variety of unlicensed spectrum for $\mathrm{WiFi}$ and Bluetooth (2.4 GHz, 3.2 GHz, $5 \mathrm{GHz}$ ), near-field communication support, and even FM. Consequently, we ignore this as a factor although very likely there will be a cost associated with specific bands as far as hardware goes (see Section $\mathrm{V}$ for one example). This cost can again be heuristically quantified as a number between 0 and 1 .

3) Interference: There are two ways in which interference can impact the fungibility scores. The interference that a band faces from adjacent bands may reduce its fungibility by reducing the useful coverage area for a given application. The restrictions on interference that may be caused by using a certain band on applications in adjacent bands may reduce the usability of a certain spectrum for certain applications. The former example is considered in more detail in Section V. The Lightsquared case alluded to previously in the paper is an example of the latter. Clearly, it is possible to use the appropriate propagation models, receiver characteristics, etc. to quantify this effect. As this is not the goal of the paper, we again use a heuristic metric between 0 and 1 (we show one calculation of this number for the $700 \mathrm{MHz}$ bands) to quantify this effect. Additionally, there are possibilities for overcoming interference (see a technical example in [22] for a fairly sophisticated scenario), again at a cost to a buyer that needs to be quantified appropriately in the fungibility score.

d) Regulatory and Miscellaneous: We adopt a heuristic metric here (in our examples, we use the extremes of 1 and 0 for the probabilistic score and distance scores respectively) as this is not easy to quantify in a general setting like the technological dimension. In the illustrative case study below, we show some sample heuristic scores.

\section{B. Computing the Overall Fungibility Score}

Given two slices of spectrum A and B, the fungibility score can be computed as follows. Let $\mathcal{D}$ be the set of dimensions under consideration. For example, $\mathcal{D}=\{$ Spatial, Temporal, Duplexing, Interference, Regulatory $\}$. Then:

$$
\begin{aligned}
P_{A, B} & =\prod_{i \in \mathcal{D}} P_{i} \\
D_{A, B} & =\sum_{i \in \mathcal{D}} D_{i}
\end{aligned}
$$

Here, we assume that the dimensions are independent in determining the overall probabilistic score $P_{A, B}$ and that the dimensions are equally important (weight $=1$ for all dimensions) in computing $D_{A, B}$ although there is an inherent normalization of distances to be between 0 and 1 . The overall fungibility scores are fairly simple and could certainly be improved with better models for these dimensions. Alternative approaches for improvement are also possible as discussed next.

\section{Improving the Fungibility Score}

Perhaps the economics alone should really determine if two spectrum bands are fungible. We could imagine a third 
party making observations over numerous buyer/seller/band tuples of successful and failed transactions and coming to probability based on that, and then modeling the components of that observation-based probability. We dont have a history of trials, so were left with estimating what the probability of a successful transaction would be based on the components that we elaborate. But outcomes also have to do with price, which would be informed by the probability - that is, a broker might negotiate price for transactions that s/he judges having a lower probability.

Regarding the distance measure, if we assume that potential spectrum buyers have to evaluate an array of spectrum offers, then they may wish to sort the offers somehow a weighted $N$-dimensional distance measure would provide an aid to this (with $N$ being the number of attributes as discussed above). The weights are determined from the buyers preferences and constraints. Taken a step further (and beyond the scope of this paper), a buyer could sort first on aggregate score. Then the buyer could sort the aggregate score AND, say technology. For example, if a buyer is using FDD, s/he would prefer a paired offer, which should show up as the closest offer; having said that, a non-paired offer could be interesting if (1) another non-paired offer existed with a sufficient frequency offset or (2) it could be used to augment a downstream channel for asymmetric capacity networks. The latter could be worked out by this kind of multilevel sort strategy.

In our ongoing and future work, we are looking at agentbased simulations with more accurate models for spatial, temporal and technological factors with embedded economic models to evaluate the simple fungibility scores outlined in this paper against simulated values that brokers or buyers may arrive at through their evaluation process.

\section{Illustrative Case Studies}

To keep things simple, in this paper we will assume that the spectrum slice is available for the duration of the secondary application (i.e., $P_{\text {temporal }}=1, D_{\text {temporal }}=0$ ).

\section{A. The $700 \mathrm{MHz}$ Bands}

We will first consider an example in the $700 \mathrm{MHz}$ bands [23] that were freed from broadcast television and are now licensed to cellular service providers to demonstrate the application of the fungibility score. Some background material is discussed first. The reasons for picking blocks within these bands for computation of the fungibility scores are that (a) they mostly eliminate the problems of coverage (spatial dimension) and frequency tunability, making the interference dimension important and (b) there has recently been significant interest in trading these bands for other bands.

Figure 2 shows the so-called lower $700 \mathrm{MHz}$ bands that range from $698 \mathrm{MHz}$ to $746 \mathrm{MHz}$. The spectrum is broken up into several blocks labeled $A$ through $E$. Blocks labeled $A$, $B, C$ are paired, i.e., they can be used in services that employ FDD, while the blocks labeled D, E are unpaired. Each of these blocks is $6 \mathrm{MHz}$ wide. Adjacent to the lower block labeled A is Channel 51 (also $6 \mathrm{MHz}$ wide) that is assigned to broadcast

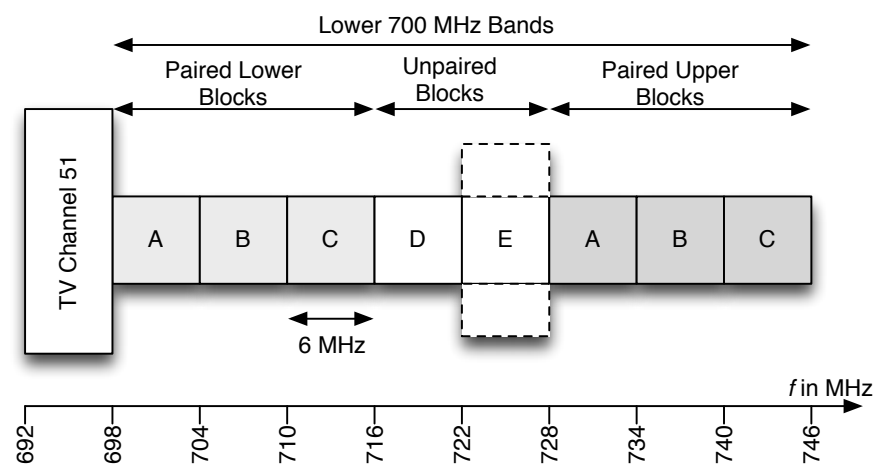

Fig. 2. The Lower $700 \mathrm{MHz}$ Bands

television. A TV tower could potentially broadcast a signal in this band with transmit power of $1000 \mathrm{~kW}$ [23]. The paired upper block labeled $A$ is adjacent to the unpaired block labeled E where a transmitter could operate at a power that could be as high as $50 \mathrm{~kW}$.

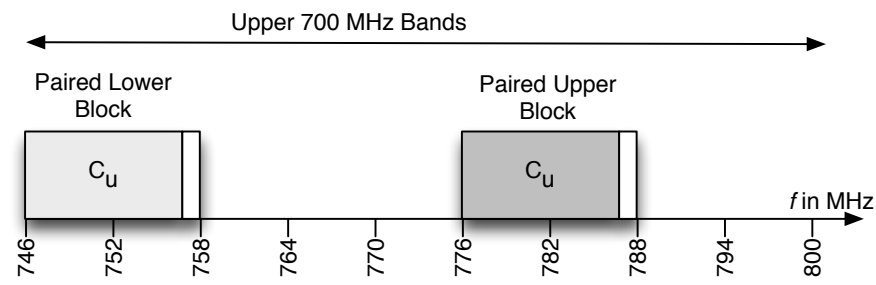

Fig. 3. The Upper $700 \mathrm{MHz}$ Bands

Figure 3 shows the so-called upper $700 \mathrm{MHz}$ bands. We do not show all of the spectrum allocated here, but focus on the paired blocks labeled $C_{u}$. The FCC actually labels them also as $\mathrm{C}$, but we have renamed them to avoid confusion with the lower C blocks. The $C_{u}$ blocks are each $11 \mathrm{MHz}$ wide.

1) First Scenario: Let us consider the scenario where a service provider has to make a choice between two sets of spectrum that are $12 \mathrm{MHz}$ wide. The preferred slice corresponds to the paired B bands in the lower $700 \mathrm{MHz}$. The available alternatives are as follows:

- Set I: Paired A bands in the lower $700 \mathrm{MHz}$

- Set II: Unpaired bands D, E

The service provider computes the fungibility scores for these two sets as follows. For both Sets I and II, $P_{\text {spatial }} \approx 1$ since the differences in frequencies are negligible. Heuristically, for Set I, $P_{\text {duplexing }}=1, D_{\text {duplexing }}=1$, since the A blocks come as a pair. For Set II, $P_{\text {duplexing }}=0.5$, $D_{\text {duplexing }}=0.5$. When interference considerations are taken into account, things get complicated. Clearly, the D,E blocks face interference from the lower $C$ and upper $A$ blocks which is much smaller than the interference that may be faced by the A blocks from Channel 51 transmissions or the $\mathrm{E}$ block transmissions. Let us assume that the D,E blocks face negligible interference (i.e., $P_{\text {interference }}=1, D_{\text {interference }}=0$ for Set II).

Let us consider Set I and the interference, only from Channel 51. In a very simplistic worst-case evaluation, one may 


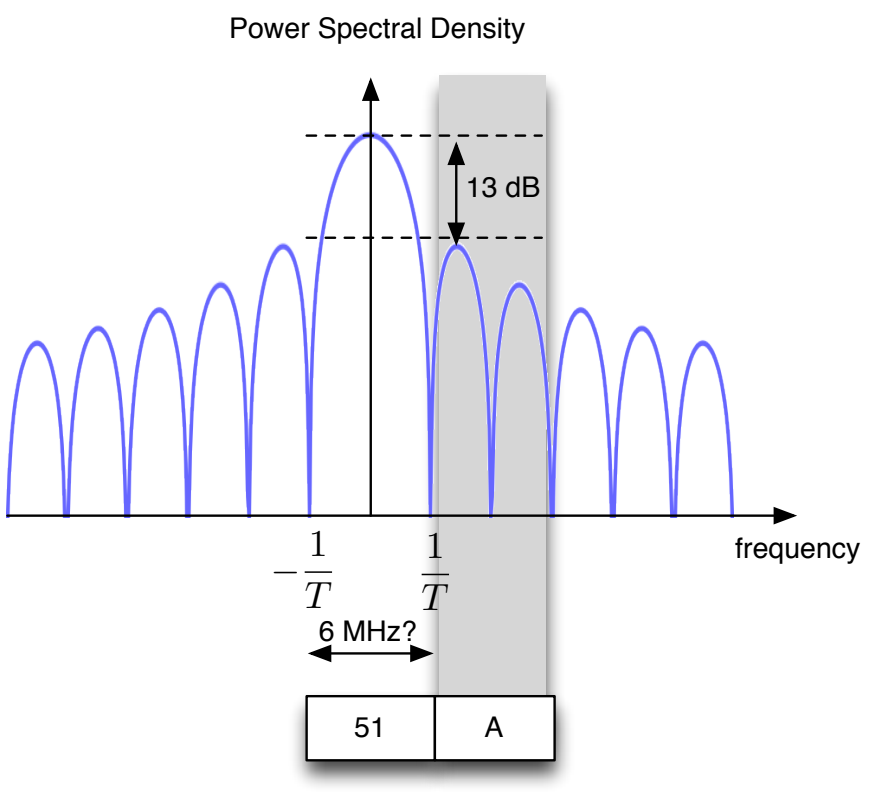

Fig. 4. Worst Case Interference from Channel 51

assume that the Channel 51 transmissions use a rectangular pulse shape which results in the classic $\operatorname{sinc} c^{2}$ power spectral density shown in Figure 4. If the $6 \mathrm{MHz}$ band includes only the main-lobe, the first dominant side-lobe is only $13 \mathrm{~dB}$ smaller than the main lobe. With a transmit power of $1000 \mathrm{~kW}$ (= $90 \mathrm{dBm}$ ), the interfering signal starts at $77 \mathrm{dBm}$. Assuming additional losses totaling $100 \mathrm{~dB}$ with geographical distances and other factors, the perceived interference could still be as high as $-23 \mathrm{dBm}{ }^{1}$. This can reduce the coverage in the lower A block by a significant factor. Without considering a full-blown analysis with specific geographical locations of the TV transmitter, the service provider's transmitter, and service provider's receivers, let us simply say that the probabilistic score is $P_{\text {interference }}=p_{i}$ and the distance score is $D_{\text {interference }}=d_{i}$. The overall fungibility scores for the two sets will be:

$$
\begin{aligned}
P_{I} & =P_{\text {duplexing }} \times P_{\text {interference }}=p_{i} \\
D_{I} & =D_{\text {duplexing }}+D_{\text {interference }}=d_{i} \\
P_{I I} & =0.5 \\
D_{I I} & =0.5
\end{aligned}
$$

We have intentionally kept the scores simple and identical (although this will not be the case with better models and more data). The idea here is that the service provider will pick Set I over Set II only if $p_{i}>0.5$ or $d_{i}<0.5$. This also assumes that the two sets have identical prices and that frequency tunability is not a problem since the frequencies are close.

Finally, the hardware costs for operating in the lower A bands are likely to be larger in reality because most operators

\footnotetext{
${ }^{1}$ Of course, this is unlikely since better pulse shapes and filters are likely to be employed by the TV transmission to contain its bandwidth and the transmit power may not be as high either.
}

preferring the lower $\mathrm{B}$ and $\mathrm{C}$ bands are proposing to use a separate band class for these bands [23]. If smartphones or other devices are not built to operate in the lower A bands, even if these bands face little interference in select geographical areas, they may be unattractive to a potential secondary user. We have not quantified this effect here.

\section{B. The $700 \mathrm{MHz}$ Bands and the $1.7 \mathrm{GHz}$ AWS Bands}

As a second illustrative example, we consider the AWS-1 paired bands [24] where the uplink is expected to use the 1700 $\mathrm{MHz}$ bands and the downlink, the $2100 \mathrm{MHz}$ bands as shown in Figure 5. Here, the preferred spectrum corresponds to the lower B and C bands (total of $24 \mathrm{MHz}-12 \mathrm{MHz}$ each for the uplink and downlink). The alternatives are:

- Set I: Paired $C_{u}$ bands in the upper $700 \mathrm{MHz}$

- Set II: Paired B bands in the AWS-1 Spectrum (see Figure 5)

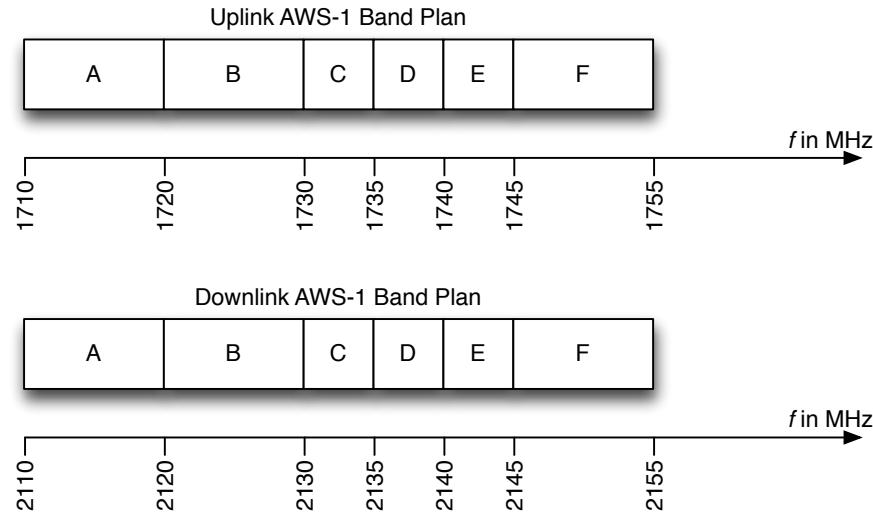

Fig. 5. The AWS-1 Spectrum

We observe three differences between the previous case study and this case study. First, duplexing is not a problem since the alternatives come in pairs. Interference to/from neighbors is also not a significant problem since the purpose of the slices of spectrum being considered are similar and they are expected to have similar protection from neighboring transmissions. Second, the previous assumption we had made about the bandwidths being identical does not apply here. The preferred spectrum has a bandwidth of $24 \mathrm{MHz}$, while Set I has $22 \mathrm{MHz}$ and Set II has $20 \mathrm{MHz}$. Finally, the spatial dimension becomes important. While it is reasonable to assume that the $C_{u}$ bands have similar coverage compared to the preferred lower $B$ and $C$ bands in the $700 \mathrm{MHz}$ spectrum, this is unlikely with the AWS-1 spectrum. Once again, using only appropriate fractions ${ }^{2}$ for calculating the fungibility scores, we arrive at (using approximations involving only the uplink AWS-1

\footnotetext{
${ }^{2} \mathrm{We}$ assume that $f_{1}=700 \mathrm{MHz}$ and $f_{2}=1700 \mathrm{MHz}$, the transmit power is $4 \mathrm{~W}$, and the receiver sensitivity is $-80 \mathrm{dBm}$ to compute the distance score using the free space loss. The distance score in this case turns out to be 0.59 .
} 
spectrum):

$$
\begin{aligned}
P_{I} & =\frac{11}{12}=0.91 \\
D_{I} & =\frac{1}{12}=0.08 \\
P_{I I} & =\frac{5}{6} \times \frac{7}{17}=0.34 \\
D_{I I} & =\frac{1}{6}+0.59=0.76
\end{aligned}
$$

Once again, very simple assumptions and models have been used which seem to imply that the $C_{u}$ bands are more fungible than the AWS-1 bands, but perhaps not significantly, if the costs are lower for acquiring access to the AWS-1 bands.

\section{Conclusions And Consequences}

Fungibility is a common assumption for market-based spectrum management. In this paper, we explored the dimensions of practical fungibility from the point of view of the spectrum buyer (which, we assert, is the relevant one). The complexity of a fungibility determination described above means that analyses dependent on this assumption should be reexamined.

Fundamentally, notions of fungibility, as with spectrum trading, are built on a licensing regime that is neutral to the technologies and services that providers used. As regulators increasingly migrate from a transmitter to a receiver perspective [25], for instance, a service provider would have to use the spectrum in a manner that did not cause interference with neighbors. Thus, the buyer of a spectrum band may still have to substantially alter the network (e.g., turn off base stations to alter the deployment) that may render a spectrum band not worth the cost.

It is also important to note that emergent technology trends can have a large influence on fungibility, so it is important not to consider it as a static notion in the realm of spectrum. For example, the idea of small cells primarily for capacity (as distinct from coverage) is emerging. In such a scenario, a buyer may engineer a system with the same cell size for different frequencies, so the weight of the spatial dimension may be lower in the future. In another example, there is a recent interest in the convergence of TDD and FDD systems, which may reduce the weight of the duplexing dimension.

Finally, we focused on "naked" spectrum trading, though this need not be the case in future. Forde et.al. [26] discuss some possibilities for how the network operator business models might evolve. For a variety of economic reasons one of the suggestions focuses on the concept of the "cellular cloud". In such a system every operator becomes some kind of virtual operator but not as we currently understand that term to mean. In this conceptualization, the physical network will be owned by some collection of entities (or indeed random individuals). An operator will be an entity that combines physical resources (base stations, back haul, spectrum etc) with applications and content to make a service offering to potential customers. In this model the network is "performed" into existence by the operator to provide services to the end user. Spectrum in this model is just one of a number of the physical resources that needs to be procured either temporarily or for longer periods of time. The fungibility metric can play a very useful role in that procurement process. It allows this future operator to for example to decide which chunks of spectrum combine with which physical infrastructure options. It would allow the operator to identify which bands can be used or combined etc, allowing the operator decide how s/he will bid. In a somewhat similar fashion, Lehr and Chapin [27] argued that, in future, the small cell architecture would cause infrastructure to concentrate (economically), which would mean that competition would have to exist at the service level (if at all).

While there are numerous technical and policy challenges associated with these possible futures, from the perspective of this paper, it is also worth considering how fungibility might apply. When infrastructure and frequency bands can be assumed, then service providers do not trade spectrum access but rather for connectivity and reachability at a particular QoS. The notion of "fungibility" then becomes less about the parameters we discussed in this paper than about capabilities that various infrastructures may offer. As in the case of "naked" spectrum, fungibility in this sense may also not be all-or-nothing. So, similar thinking about the substitutability of complex, multidimensional objects would apply, even if the determinants of these are different.

The preliminary work presented in this paper suggests a richer followup studies. Such studies should include more careful and detailed technical models, modeling user behavior (using tools such as agent-based computational economics, as was demonstrated by [28]) and revisiting prior studies (e.g., [1]) to assess the impact of fungibility scores on spectrum trading systems. It also suggests that a focus on "naked" spectrum may be too myopic - that a broader, service level notion of fungibility should be developed in parallel.

\section{ACKNOWLEDGMENT}

This work was supported in part by the U.S. National Science Foundation under Grant 1149422.

\section{REFERENCES}

[1] C. Caicedo and M. B. Weiss, "The viability of spectrum trading markets," IEEE Communications Magazine, vol. 43, no. 3, pp. 46-52, 2011.

[2] R. Akalu, "Why there have been so few secondary trades in the UK: lessons for Europe," info, vol. 12, no. 1, pp. 10-17, 2010.

[3] L. Doyle and T. Forde, "Towards a fluid spectrum market for exclusive usage rights," in IEEE DySPAN, 2007, pp. 620-632.

[4] E. Kwerel and J. Williams, "A proposal for a rapid transition to market allocation of spectrum," OPP Working Paper Series 38, 2002.

[5] L. McKnight, R. Linsenmayer, and W. Lehr, "Best effort versus spectrum markets: Wideband and Wi-Fi versus 3G MVNOs?" in Telecommunications Policy Research Conference, 2001.

[6] P. Xavier and D. Ypsilanti, "Policy issues in spectrum trading," info, vol. 8, no. 2, pp. 34-61, 2006.

[7] A. Kerans, D. Vo, P. Conder, and S. Krusevac, "The price of spectrum based on physical criteria," in Symposium on New Frontiers in Dynamic Spectrum Access Networks (DySPAN). IEEE, 2011.

[8] R. J. Teweles, F. J. Jones, and B. Warwick, The futures game : who wins? who loses? and why?, 3rd ed. New York: McGraw-Hill, 1999.

[9] O. E. Williamson, Markets and Hierarchies. New York: Free Press, 1975. 
[10] P. J. Weiser and D. Hatfield, "Spectrum policy reform and the next frontier of property rights," George Mason Law Review, vol. 15, no. 3, pp. 549-609, 2008.

[11] T. W. Hazlett, "A law \& economics approach to spectrum property rights: A response to Weiser and Hatfield," George Mason Law Review, vol. 15, no. 4, pp. $975-1023,2008$

[12] M. B. Weiss and L. Cui, "Spectrum trading with interference rights," in International Conference on Cognitive Radio Oriented Wireless Networks (CROWNCOM), 2012.

[13] J. W. Mayo and S. Wallsten, "Enabling efficient wireless communications: The role of secondary spectrum markets," Information Economics and Policy, vol. 22, no. 1, pp. 61-72, 2010.

[14] R. J. Akalu and A. D. Arias, "Assessing the policy of spectrum trading in the UK," Info, vol. 14, no. 1, 2012.

[15] T. Hazlett, "Creating efficient spectrum property," Spectrum Markets: Challenges Ahead Workshop, Evanston, IL, June 2011.

[16] D. Tipper, P. Krishnamurthy, A. Rezgui, and P. Pacharintanakul, "Dimming cellular networks," IEEE Globecom, 2010.

[17] M. B. Weiss, M. Altamimi, and L. Cui, "Spatio-temporal spectrum modeling: Taxonomy and economic evaluation of context acquisition," Telecommunications Policy, vol. 36, no. 4, pp. 335 - 348, 2012.

[18] P. W. Chan, E. S. Lo, R. R. Wang, E. K. Au, V. K. Lau, R. S. Cheng, W. H. Mow, R. D. Murch, and K. B. Letaief, "The evolution path of 4G networks: FDD or TDD?" IEEE Communications Magazine, vol. 44, no. 12 , pp. 42-50, 2006.

[19] H. Martikainen, "Analysis of duplexing modes in the IEEE 802.16 wireless system," pp. 849-856, 12-15 April, 20102010.

[20] P. Clarke, "Lightsquared: a continuing threat to GNSS?" Astronomy \& Geophysics, October 2011.

[21] Broadcom's new combo chip integrates $802.11 \mathrm{n} \mathrm{Wi-Fi} \AA$, bluetooth $\mathbb{R}$ and $\mathrm{fm}$ to bring multimedia applications to mobile devices. [Online]. Available: http://www.broadcom.com/press/release.php?id=1233460

[22] J. G. Andrews, W. Choi, and R. Heath, "Overcoming interference in spatial multiplexing MIMO cellular networks," IEEE Wireless Communications, December 2007.

[23] FCC, "Notice of proposed rulemaking in the matter of promoting interoperability in the $700 \mathrm{MHz}$ commercial spectrum," FCC 12-13, 2012.

[24] "AWS-1 band plan." [Online]. Available: http://wireless.fcc.gov/services/aws/data/awsbandplan.pdf

[25] J. P. de Vries and K. A. Sieh, "Reception-oriented radio rights: Increasing the value of wireless by explicitly defining and delegating radio operating rights," Telecommunications Policy, 2012.

[26] T. K. Forde, I. Macaluso, and L. E. Doyle, "Exclusive sharing and the virtualization of the cellular network," 2011.

[27] J. Chapin and W. Lehr, "Mobile broadband growth, spectrum scarcity, and sustainable competition," in Telecommunications Policy Research Conference, 2011.

[28] A. Tonmukayakul and M. B. Weiss, "A study of secondary spectrum use using agent-based computational economics," Netnomics, vol. 9, no. 2, pp. 125-151, 2008. 